\title{
Accurate Lattice Energies for Molecular Crystals from Experimental Crystal Structures
}

Sajesh P. Thomas, ${ }^{t s}$ Peter R. Spackman, Dylan Jayatilaka and Mark A. Spackman"

$\dagger$ School of Molecular Sciences, University of Western Australia, Perth 6009, Australia

\begin{abstract}
Using four different benchmark sets of molecular crystals we establish the level of confidence for lattice energies estimated using CE-B3LYP model energies and experimental crystal structures. [See IUCrJ, 2017, 4, 575-587.] We conclude that they compare very well with available benchmark estimates derived from sublimation enthalpies, and in many cases they are comparable with - and sometimes better than - more computationally-demanding approaches, such as those based on periodic DFT plus dispersion methodologies. The performance over the complete set of 110 crystals indicates a mean absolute deviation from benchmark energies of only $6.6 \mathrm{~kJ} \mathrm{~mol}^{-1}$. Applications to polymorphic crystals and larger molecules are also presented and critically discussed. The results highlight the importance of recognizing the consequences of different sets of crystal/molecule geometries when comparing different methodologies, as well as the need for more extensive benchmark sets of crystal structures and associated lattice energies.
\end{abstract}




\section{INTRODUCTION}

The calculation of lattice energies for molecular crystals is central to modern first-principles approaches to the prediction and rationalisation of their structure and important chemical and physical properties. As a consequence, the computation of organic crystal lattice energies with "chemical accuracy" has received increased attention in recent years (up to date summaries of the literature can be found in several recent reviews'). For some time now our own research has focused on a multi-faceted approach to enhancing our understanding of the interactions between molecules and their packing in crystals, principally through the development of new computational and graphical tools that can be readily applied to the analysis of experimental crystal structures. Elements of this approach include the now commonplace Hirshfeld surface analysis, ${ }^{2}$ characterisation of void space in molecular crystals, ${ }^{3}$ color mapping on molecular surfaces of properties derived from molecular wavefunctions, ${ }^{4}$ and the calculation of remarkably accurate model intermolecular interaction energies, in particular the CE-B3LYP model energies, along with a graphical representation of their magnitude in the form of 'energy frameworks'. ${ }^{6}$ We have demonstrated in recent work how this combined approach considerably enhances the understanding of the nature of intermolecular interactions in the context of the packing of molecules in crystals, in particular their relationship with bulk mechanical properties, , tasas.s. $^{\text {and }}$ and the nature of host-guest interactions in clathrates of Dianin's compound and of hydroquinone. ${ }^{2}$ In the course of that work we reported lattice energies for crystals of formamide, $s$-triazine, hexachlorobenzene, hexabromobenzene, $\mathrm{Cr}(\mathrm{CO})_{6}$ and $\mathrm{VO}(\mathrm{acac})_{2}$, obtained from a converged sum

of pairwise CE-B3LYP model interaction energies. Those results compared surprisingly well with experimental sublimation enthalpies ${ }^{10}$ or with values obtained by Gavezzotti's PIXEL method," prompting us to assess the reliability of these lattice energies applied to a more extensive set of crystal structures.

Here we present a comprehensive, detailed and critical assessment of the performance of CEB3LYP lattice energies in comparison with a variety of state of the art computational approaches for molecular crystals, using several recently described databases of benchmark experimental 
lattice energies, and materials ranging from noble gases to large organic molecules such as coronene and rubrene. Our principal goal is to establish the level of confidence that can be expected for lattice energies computed with the CE-B3LYP model, as well as highlight the way in which it can provide useful and reliable estimates for much larger molecular materials than are

currently tractable with more rigorous computational approaches. In the process we identify inconsistencies with some current benchmark data, leading us to make some recommendations for future work of this kind.

\section{COMPUTATIONAL METHODS}

It is important to give some perspective on the computational approaches, methodologies and benchmarks that are relevant to our goal of making a critical comparison of CE-B3LYP model lattice energies with the results from more rigorous computational approaches. We identify four broad computational strategies to predicting lattice energies:

- $A b$ initio prediction of crystal structure with one level of theory, including space group, cell parameters and atomic coordinates; lattice energy computed at the same - or different - level of theory.

- Starting from an experimental crystal structure, cell parameters and atomic coordinates are optimized with one level of theory; lattice energy computed at the same - or different - level of theory.

- Using known space group and fixed experimental cell parameters, atomic coordinates are optimized with one level of theory; lattice energy computed at the same - or different - level of theory.

- Lattice energy computed using experimental crystal structures, typically with bond lengths to $\mathrm{H}$ atoms adjusted to standard neutron values."2 This is the strategy pursued in the present work. It should be immediately evident that any attempt at a systematic comparison with, or between, published lattice energies is not straightforward. Almost every set of published lattice energies is based on a different set of crystal structures, with the result that differences between 
lattice energies are almost always intertwined with differences between chosen geometries. Benchmark values for "experimental" lattice energies are typically based on measured sublimation enthalpies $^{10}$ (or, less frequently, vaporization and fusion enthalpies combined with heat capacities ${ }^{13}$, and for organic molecular crystals these are generally acknowledged to have an inherent uncertainty of $\sim 5 \mathrm{~kJ} \mathrm{~mol}^{-1} .^{14}$ Deriving lattice energies from sublimation enthalpies must take account of the magnitude (and sign) of the energetic or thermodynamic quantities that are implicitly contained in the two quantities. This is typically expressed by a relationship of the form: ${ }^{15}$

$$
\begin{aligned}
\Delta H_{\mathrm{sub}}(T) & =\left(E_{\mathrm{el}}^{\mathrm{g}}+E_{\mathrm{trans}}^{\mathrm{g}}+E_{\mathrm{rot}}^{\mathrm{g}}+E_{\mathrm{vib}}^{\mathrm{g}}\right)-\left(E_{\mathrm{el}}^{\mathrm{s}}+E_{\mathrm{vib}}^{\mathrm{s}}\right)+p V \\
& =\left(E_{\mathrm{el}}^{\mathrm{g}}-E_{\mathrm{el}}^{\mathrm{s}}\right)+\left(E_{\mathrm{vib}}^{\mathrm{g}}-E_{\mathrm{vib}}^{\mathrm{s}}\right)+4 R T \\
& =\Delta E_{\mathrm{el}}+\Delta E_{\mathrm{vib}}+4 R T \\
& =-E_{\mathrm{lat}}+\Delta E_{\mathrm{vib}}+4 R T
\end{aligned}
$$

where ideal gas behavior is assumed, and the last term is $3.5 R T$ for linear molecules and $3 R T$ for atoms. (It is worthwhile noting here that eq. (1) defines the lattice energy, $E_{\text {lat }}$, to be a negative quantity. Although this convention is used throughout the present work it is not universal; e.g., Otero-de-la-Roza and Johnson tabulated positive lattice energies for the C21 crystals $^{1 s_{a}}$ ). Several approaches have been used to obtain "experimental" benchmark lattice energies by estimating the thermal effects in eq. (1), $\Delta E_{\mathrm{vib}}+4 R T$, at different levels of sophistication. The most common approximates these two terms by $-2 R T$ ( $-1.5 R T$ for linear molecules), a result that assumes no difference between gas and crystal intramolecular vibrations, and the intermolecular vibrational energy is at the high-temperature limit of $6 R T$. These and other assumptions underlying this approximation are discussed in detail in several places. ${ }^{15, \mathrm{~b}, 16}$ As argued by Maschio et al.," in some situations the $2 R T$ correction "introduces perhaps more uncertainty than accuracy", as the neglected terms are often of the same order of magnitude as the experimental uncertainty in $\Delta H_{\text {sub }}(T)$. More sophisticated approaches to estimating the thermal effects in eq. (1) have been reported ${ }^{15, \mathrm{~b}}$ and these have contributed to the popularity of the X23 benchmark set of experimental lattice energies. 
2.1. Benchmark data sets. For the present purposes we compare lattice energies computed by a converged sum of pairwise CE-B3LYP model energies with 'experimental' lattice energies derived from observed thermodynamic data, for the following four benchmark sets:

X23 set. The original C21 set, ${ }^{\text {,s }}$ with hexamine (hexamethylenetetramine) and succinic acid added. ${ }^{\text {ss }}$ Those publications reported different estimates of $\Delta E_{\text {vib }}+4 R T$, as well as using different $\Delta H_{\text {sub }}(T)$ values for anthracene, and as a consequence the mean absolute deviation between the two estimates of $\mathrm{C} 21$ benchmark energies is $2.3 \mathrm{~kJ} \mathrm{~mol}^{-1}$, with a maximum deviation of $12.1 \mathrm{~kJ} \mathrm{~mol}^{-1}$. For our purposes we use the $\mathrm{X} 23$ benchmark 'experimental' lattice energies estimated by Reilly and Tkatchenko ${ }^{\text {ss }}$ with updated values for benzene, naphthalene and cytosine (see Supporting Information Table S1). The average benchmark lattice energy for the X23 set is $-85.1 \mathrm{~kJ} \mathrm{~mol}^{-1}$.

G60 set. This set of molecular crystals was originally compiled by Maschio et al.," and contains "a wide range of chemical functionalities", more than one-third of which are either chloro or nitro substituents. In that work computed lattice energies were compared directly with experimental sublimation enthalpies. More recently Cutini et al. ${ }^{\text {ss }}$ corrected those sublimation enthalpies by a constant factor of $2 R T$ (i.e. $E_{\text {lat }}=-\Delta H_{\text {sub }}-2 R T$ ), and used those lattice energy estimates in their assessment of several computational methods. In what follows we use the experimental lattice energy estimates from Cutini et al. ${ }^{\text {se }}$ as benchmark values for the G60 set (Table S2). The average benchmark lattice energy for the $\mathrm{G} 60$ set is $-102.9 \mathrm{~kJ} \mathrm{~mol}^{-1}$.

K7 set. Cutini and co-workers also compiled a smaller set of just seven molecular crystals: acetamide, two polymorphs of acetylene, boric acid, $\mathrm{HCN}$, ice XI and propane, with lattice energies estimated as for the G60 set, and we use those values in our own benchmarking (Table $\mathrm{S} 3)$. The average benchmark lattice energy for the $\mathrm{K} 7$ set is $-53.2 \mathrm{~kJ} \mathrm{~mol}^{-1}$.

Z20 set. In their studies of $\operatorname{CCSD}(\mathrm{T}) / \mathrm{CBS}$ fragment-based calculations to predict lattice energies and sublimation enthalpies for molecular crystals, Červinka et al. compiled another set of 25 molecular crystals that includes noble gases, nonpolar hydrocarbons, $\mathrm{N}_{2}, \mathrm{~F}_{2}, \mathrm{CO}_{2}$, and a 
number of hydrogen-bonded crystals. ${ }^{13}$ Experimental lattice energies for benchmarking purposes (Table S4) have been obtained from experimental sublimation enthalpies adjusted to $0 \mathrm{~K}$, corrected for relaxation and zero-point energies, as described in those publications. For various reasons this procedure was not feasible for methane, formaldehyde, ethanol and acetone, and it was also evident that the crystal structure used for hydrazine in that work contained errors (see Table S4). As a consequence only 20 of the 25 crystal structures of this set, that we have designated Z20, have been used in the present analysis. The average benchmark lattice energy for the $\mathrm{Z} 20$ set is $-41.7 \mathrm{~kJ} \mathrm{~mol}^{-1}$.

These four benchmark sets contain a total of 101 different materials. Acetic acid and formamide are common to X23, G60 and Z20, anthracene, benzene, imidazole, naphthalene, $\beta$ oxalic acid and urea are common to X23 and G60, formic acid is common to G60 and Z20, ammonia and $\mathrm{CO}_{2}$ are common to both $\mathrm{X} 23$ and $\mathrm{Z} 20$, and both $\mathrm{K} 7$ and Z20 include propane. However, because the benchmark lattice energies have been obtained from experimental data in several different ways, and because different crystal structures at different temperatures have often been used for the same compound in separate benchmark sets (e.g., ACETAC01 and ACETAC05, BENZEN and BENZEN07, UREAXX and UREAXX09), we include calculations on duplicate crystal structures in our analyses that follow. This results in a combined set of 110 CE-B3LYP lattice energies, which includes two polymorphic pairs (oxalic acid and acetylene), but conspicuous by their absence are organic molecules containing F, Br, I, P, S or Se, or any metal-organic compounds.

2.2. The CE-B3LYP energy mode1. Our CE-B3LYP model energies have been described in detail elsewhere, but it is worthwhile providing an outline here. Originally devised for organic molecular crystals,,$^{\text {sb }}$ it is based on quantum mechanical charge distributions for unperturbed monomers, and has recently been shown to apply equally well to molecular crystals comprising metal coordination compounds, organic salts, solvates and open shell molecules (radicals). ${ }^{\text {sa }}$ The breakdown of the total interaction energy into electrostatic, polarization, dispersion and exchange-repulsion terms, 


$$
\begin{aligned}
E_{\mathrm{tot}} & =E_{\mathrm{ele}}+E_{\mathrm{pol}}+E_{\mathrm{dis}}+E_{\mathrm{rep}} \\
& =k_{\mathrm{ele}} E_{\mathrm{ele}}^{\prime}+k_{\mathrm{pol}} E_{\mathrm{pol}}^{\prime}+k_{\mathrm{dis}} E_{\mathrm{dis}}^{\prime}+k_{\mathrm{rep}} E_{\mathrm{rep}}^{\prime}
\end{aligned}
$$

is not new, and dates back to energy decomposition methods via variational ${ }^{18}$ and perturbation based $^{19}$ approaches. In our model $E_{\text {ele }}^{\prime}$ is the electrostatic interaction energy between monomer charge distributions, and $E_{\text {rep }}^{\prime}$, the exchange-repulsion energy, both derived from the antisymmetrized product of $\mathrm{B} 3 \mathrm{LYP} / 6-31 \mathrm{G}(\mathrm{d}, \mathrm{p})$ monomer spin orbitals. ${ }^{20}$ The polarization energy term, $E_{\mathrm{pol}}^{\prime}$, is a simple sum of terms of the kind $-1 / 2 \alpha|F|^{2}$, where $\alpha$ are isotropic polarizabilities for atoms $^{21}$ or monatomic ions ${ }^{s_{1}}$ and the electric field $F$ is computed at each atomic nucleus from the B3LYP/6-31G(d,p) charge distribution of the other monomer. $E_{\text {dis }}^{\prime}$ is Grimme's D2 dispersion correction $^{22}$ summed over all intermolecular atom pairs. Optimum values of the scale factors $\boldsymbol{K}_{\mathrm{cl}}$, etc. in eq. (2) have been determined by calibration against counterpoise-corrected B3LYP-D2/6$31 \mathrm{G}(\mathrm{d}, \mathrm{p})$ interaction energies for 1,794 molecule/ion pairs extracted from 171 crystal structures. The mean absolute deviation (MAD) of these CE-B3LYP model energies from the DFT benchmark values is $2.4 \mathrm{~kJ} \mathrm{~mol}^{-1}$ for pairwise energies that span a range of $3.75 \mathrm{MJ} \mathrm{mol}^{-1} .^{\text {sa }}$

Although our approach has much in common with Gavezzotti's PIXEL method," which has become popular in applications to organic molecular crystals, there are many significant differences between the two. PIXEL employs a fine-grained discrete representation of the molecular electron density as a sum of charged volume elements. The electrostatic energy between molecular charge densities is fundamentally the same in the two methods (although PIXEL commonly uses MP2/6-31G(d,p) monomer electron densities). All other terms in the PIXEL approach make use of the same discrete representation of the electron density, and incorporate a set of atomic polarizabilities, as well as adjustable parameters to account for short separations, damping of dispersion energies and a scale factor and power law dependence for the repulsion energy. These parameters were optimized to minimize the deviation between computed lattice energies and experimental sublimation enthalpies for a representative set of organic crystal structures. 
2.3. Calculation of lattice energies. CE-B3LYP lattice energies are computed by direct summation of interaction energies in CrystalExplorer17 $7^{23}$ over molecules B interacting with a central molecule A until $E_{\text {lat }}$ is converged to better than $1 \mathrm{~kJ} \mathrm{~mol}^{-1}$, using a cutoff based on the separation of molecular centroids, $R_{\wedge в}$. Sums over $E_{\text {dis }}$ and $E_{\text {rep }}$ converge rapidly, but the sum over $E_{\text {ele }}$ terms is much longer-range, with highly dipolar compounds (e.g., HCN, formamide, zwitterionic amino acids) requiring much greater cutoff distances than non-dipolar molecules (e.g., anthracene, coronene). For molecules separated by more than $12 \AA E_{\text {ele }}$ is computed using a distributed multipole model of the electron density. ${ }^{24}$ As described in detail elsewhere, ${ }^{1725}$ for unit cells with non-zero dipole moments (i.e., polar space groups such as $P 2_{1}, P n a 2_{1}$ and $F d d 2$ ) the lattice summation above is not representative of the entire crystal, and an additional consideration is required. The complete term for the lattice energy is

$$
E_{\text {lat }}=\frac{1}{2} \sum_{R_{A \mathrm{~B}}<R} E_{\mathrm{tot}}^{\mathrm{AB}}+E_{\text {cell dipole }}=\frac{1}{2} \sum_{R_{\mathrm{AB}}<R} E_{\mathrm{tot}}^{\mathrm{AB}}-\frac{2 \pi p_{\text {cell }}^{2}}{3 Z V_{\text {cell }}},
$$

where the second term is the cell dipole energy correction. ${ }^{250} p_{\text {cal }}$ is the magnitude of the dipole moment of the unit cell (obtained here as the vector sum of molecular dipole moments), $V_{\text {cal }}$ its volume and $Z$ has its usual meaning. Table $\mathrm{S} 5$ provides details of this term for all molecules in polar space groups considered in this study. As noted elsewhere, small, often less than $1 \mathrm{~kJ} \mathrm{~mol}^{-1}$, but it can be substantial and essential when cell dipole moments are large (e.g., trioxane, nitroguanidine and acetamide, for which $E_{\text {cell dipole }}$ is $-7.5,-11.6$ and $7.8 \mathrm{~kJ} \mathrm{~mol}^{-1}$, respectively), and especially when combined with small unit cell volumes (e.g., HF and $\mathrm{HCN}$, where $E_{\text {cell dipole }}$ is -17.9 and $-24.6 \mathrm{~kJ} \mathrm{~mol}^{-1}$, respectively).

A further consideration is necessary when computing lattice energies by this approach (i.e. summation of pairwise interaction energies), as it implicitly assumes molecular geometries in the gas phase are unchanged from those in the crystal. Although equating $\Delta E_{\mathrm{el}}$ to $-E_{\mathrm{lat}}$ in eq. (1) is formally correct, it disguises the fact that $\Delta E_{\mathrm{el}}$ necessarily includes intramolecular energies, as 
well as the intermolecular interactions included in eq. (3). To shed some light on the missing contribution we partition the crystal electronic energy into intramolecular and intermolecular contributions, and write

$$
\begin{aligned}
\Delta E_{\mathrm{el}} & =E_{\mathrm{el}}^{\mathrm{g}}-E_{\mathrm{el}}^{\mathrm{s}} \\
& =E_{\mathrm{el}, \text { intra }}^{\mathrm{g}}-\left(E_{\mathrm{el}, \text { intra }}^{\mathrm{s}}+E_{\mathrm{el}, \text { inter }}^{\mathrm{s}}\right) \\
& =\left(E_{\mathrm{el}, \text { intra }}^{\mathrm{g}}-E_{\mathrm{el}, \text { intra }}^{\mathrm{s}}\right)-E_{\mathrm{lat}} \\
& =\Delta E_{\mathrm{relax}}-E_{\text {lat }}
\end{aligned}
$$

The molecular relaxation energy in this expression, $\Delta E_{\text {relax }}$, is the electronic energy lowering of a molecule accompanying relaxation of its geometry in the gas phase. This term is (normally) automatically taken into account in quantum mechanical studies that perform geometry optimizations for the crystal and isolated molecules, but it is not included in our lattice energy estimate, eq. (3), which can compromise comparisons between the results, or with experimental sublimation enthalpies. If we ignore the relaxation energy in our lattice energy estimates we will systematically overestimate the magnitude of the lattice energy. For molecules that are essentially rigid (i.e. possess no internal degrees of freedom) this term is usually negligible, and that consideration underlies the choice of most molecules in the benchmark sets described earlier. But there are important instances where $\Delta E_{\text {relax }}$ is large and must be accounted for, in particular oxalic acid, urea and cytosine in the X23 set. For oxalic acid the lowest energy gas phase structure features two cyclic intramolecular hydrogen bonds ${ }^{26}$ in contrast to the extended conformation in both crystal polymorphs. The $\mathrm{N}$ atoms in urea, which are planar in the crystal, are known to be pyramidal in the gas phase..$^{27}$ And for cytosine, the enol-amino forms are known to be more abundant in the gas phase than the keto-amino tautomer present in the crystal. ${ }^{28}$ We have estimated relaxation energies for molecules in these four crystal structures by performing geometry optimizations at the MP2/6-311G++(d,p) level of theory for appropriate crystal and gas-phase structures, using Gaussian09..$^{29}$ For the oxalic acid polymorphs there is also a small intramolecular energy difference for the two forms, and an estimate of this was obtained from constrained optimizations of the positions of just the $\mathrm{H}$ atoms, as described by Cruz-Cabeza and 
Bernstein..$^{30}$ The relaxation energies obtained in this manner (Table S1) are relatively crude estimates; they have the correct sign, in all cases improving agreement with benchmark experimental lattice energies, but the magnitude of the corrections depends greatly on the level of theory and basis set chosen.

\section{RESULTS AND DISCUSSION}

3.1. X23 comparisons. Details regarding the performance of a large number of computational sets of lattice energies for X23 crystals are summarized in Table 1, and Figure 1 provides a graphical comparison between the best performing of those approaches. For all sets of literature results the fit statistics have been recalculated against the revised benchmark energies in Table S1, and the mean signed deviation (MD) for each model is systematically defined here to refer to the mean of model lattice energy minus the benchmark value. For these reasons the current statistics may be slightly different from (and MD is often better than) those given in the relevant publications. We see from these results that the CE-B3LYP model performs remarkably well, with a MD near zero and a mean absolute deviation (MAD) of only $5.1 \mathrm{~kJ} \mathrm{~mol}^{-1}$, a performance of similar quality to the two B3LYP plus dispersion approaches (B3LYP-D*/TZP and SP-B3LYP-D*). Figure 1 identifies a number of trends and systematic outliers for several models. There is evidence that CE-B3LYP systematically underbinds 'van der Waals' solids

such as anthracene, benzene, naphthalene, pyrazine and triazine, possibly indicative of a limitation of the present dispersion energy model. But this behavior is also clearly evident for several other models (e.g., vdW-DF2/DZP, B3LYP-D*/TZP and SP-B3LYP-D*), most obviously for anthracene and naphthalene. Notable outliers in Figure 1 are cyanamide, urea and cytosine. For cyanamide all models in Figure 1 predict a lattice energy greater (more negative) than the benchmark value, and even the best performing model, TPSS-D3, overbinds this crystal by $\sim 11 \mathrm{~kJ} \mathrm{~mol}^{-1}(13 \%)$. A similar observation can be made for urea, suggesting that for this molecule the non-planarity of the gas-phase structure may not have been taken into account in some of the literature studies. Results for cytosine reveal that a majority of approaches significantly underbind the crystal, especially the vdW-DF and vdW-DF2 functionals, and this 
outcome will be even worse if the different tautomer in the gas phase was accounted for. In summary these results, based on experimental crystal structures, convincingly show that the present CE-B3LYP model typically yields lattice energies within $\sim 5 \mathrm{~kJ} \mathrm{~mol}^{-1}$ of the well-curated X23 benchmark results - provided the relaxation energy is estimated when appropriate.

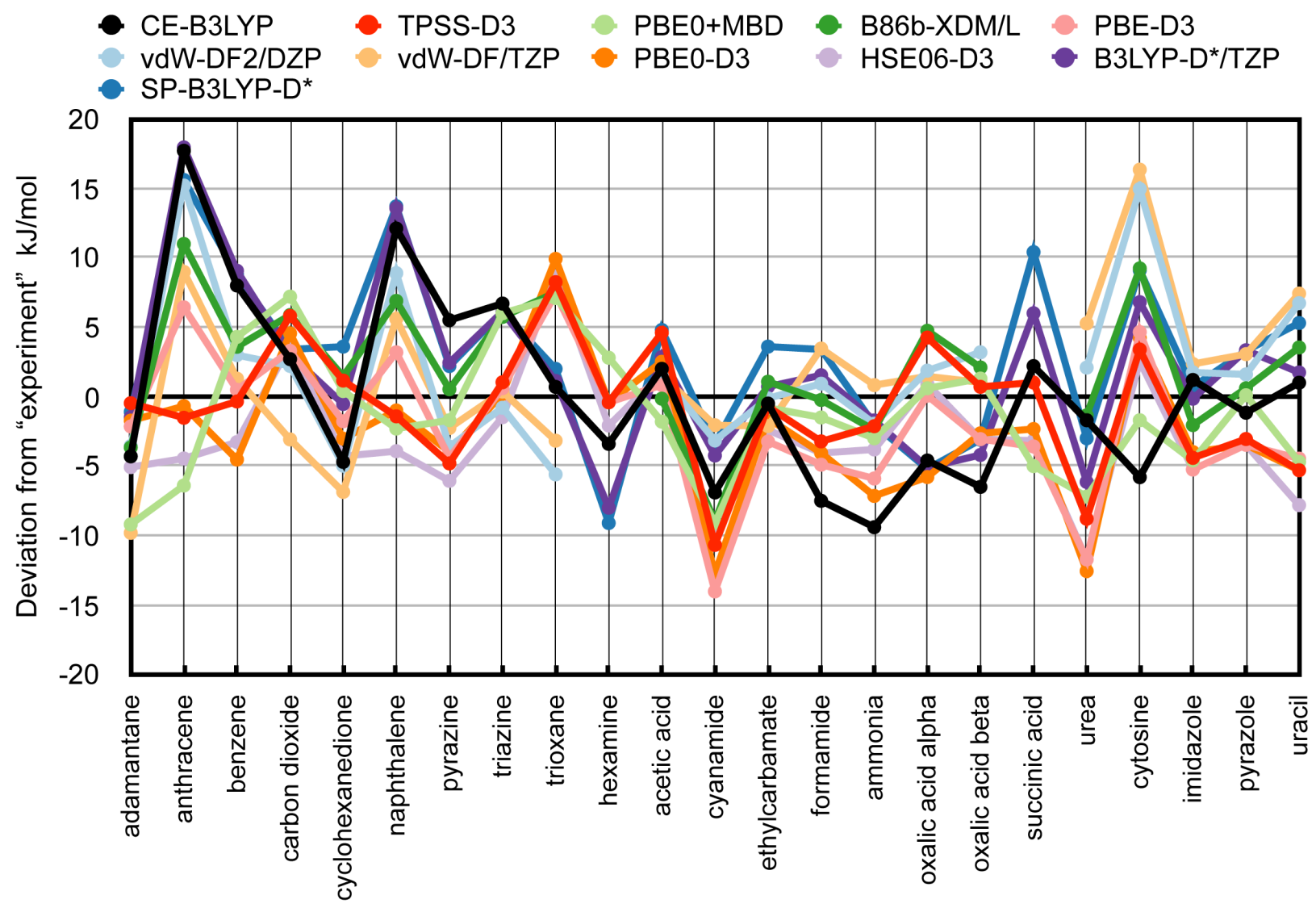

Figure 1. Deviation of computational lattice energy estimates from the X23 benchmarks. For clarity only a subset of the best performing sets of calculations from Table 1 are displayed for comparison with the performance of the CE-B3LYP model (black points and line). Positive deviations indicate that a particular model is underbinding for that structure. 
Table 1. Mean absolute deviation (MAD), mean signed deviation (MD), standard deviation from the mean (SD), all in $\mathrm{kJ} \mathrm{mol}^{-1}$, and mean absolute percent deviation (MA\%D) of computational estimates of lattice energies from the X23 benchmarks. As lattice energies are negative and MD is defined as model minus X23 benchmark value, then if MD is significantly positive the model is systematically underbinding.

\begin{tabular}{|c|c|c|c|c|}
\hline & MAD & MD & $\mathrm{SD}$ & MA\%D \\
\hline TPSS-D3 a & 3.4 & -0.7 & 4.3 & 4.7 \\
\hline PBE0-MBD & 3.9 & -1.3 & 4.6 & 5.6 \\
\hline B86b-XDM/L & 3.9 & 2.1 & 4.6 & 5.4 \\
\hline PBE-D3 a & 4.1 & -1.8 & 5.0 & 5.5 \\
\hline $\mathrm{vdW}-\mathrm{DF} 2 / \mathrm{DZP}$ & 4.2 & 1.9 & 5.5 & 4.8 \\
\hline vdW-DF/TZP & 4.2 & 1.4 & 5.5 & 4.9 \\
\hline PBE0-D3 & 4.3 & -2.4 & 4.9 & 6.0 \\
\hline HSE06-D3 。 & 4.5 & -2.9 & 4.3 & 5.9 \\
\hline B3LYP-D*/TZP & 4.7 & 1.9 & 6.0 & 5.8 \\
\hline CE-B3LYP : & 5.1 & 0.1 & 6.9 & 6.5 \\
\hline SP-B3LYP-D* & 5.3 & 3.0 & 5.9 & 6.5 \\
\hline B86b-XDM & 5.7 & 1.3 & 7.3 & 7.3 \\
\hline PBEh-3c & 6.0 & -0.1 & 7.1 & 7.9 \\
\hline PBE-MBD $^{\circ}$ & 6.0 & -4.8 & 5.1 & 7.9 \\
\hline PBE-XDM $^{*}$ & 6.4 & 2.5 & 8.1 & 7.9 \\
\hline PBE-D2 ${ }^{k}$ & 7.5 & -6.3 & 7.8 & 9.6 \\
\hline B3LYP-D3-gCP/SVP & 7.7 & -2.4 & 8.9 & 11.1 \\
\hline HF-3c ${ }^{m}$ & 8.1 & -5.6 & 8.6 & 10.6 \\
\hline LMP2/p-aug-6-31G(d,p) ${ }^{n}$ & 8.7 & 2.9 & 10.5 & 11.1 \\
\hline FIT $\circ$ & 9.1 & 7.9 & 7.7 & 10.5 \\
\hline PBE-D3-gCP/SVP & 10.0 & 4.6 & 12.3 & 14.1 \\
\hline PBE0-TS & 10.0 & -9.6 & 8.0 & 12.6 \\
\hline
\end{tabular}




\begin{tabular}{|l|c|c|c|c|}
\hline PBE-TS $^{\circ}$ & 13.5 & -13.2 & 8.2 & 17.0 \\
\hline W99rev6311P5 $^{\circ}$ & 14.1 & 13.9 & 8.5 & 15.9 \\
\hline B3LYP ${ }^{9}$ & 28.7 & 26.3 & 28.1 & 35.6 \\
\hline
\end{tabular}

a Unit cell and atomic positions fully optimized. ${ }^{31}$

b Single point energies using PBE-TS optimized crystal geometries..$^{\text {sb }}$

c C21 set only; unit cell and atomic positions fully optimized; 'large' plane wave basis set.23

d C21 set only; unit cell and atomic positions fully optimized; counterpoise corrected to account for BSSE. .32

e Single point energies using TPSS-D3 optimized crystal geometries. ${ }^{31}$

f Unit cell and atomic positions fully optimized; counterpoise corrected to account for BSSE. ${ }^{150}$

g Present work; experimental crystal structures at or near room temperature (see Table S1)

$\mathrm{h}$ Single point energies at the B3LYP-D*/TZP level of theory using dispersion-scaled HF-3c optimized geometries..$^{150}$

i C21 set only; unit cell and atomic positions fully optimized; similar results to ref. ${ }^{\text {ssc except for }}$ differences of $\sim 20 \mathrm{~kJ}$ mol-1 for oxalic acid polymorphs, suggesting rearrangement energy from crystal to gas phase has not been included. ${ }^{15 a}$

j Anthracene and naphthalene not included because SCF did not converge. ${ }^{33}$

$\mathrm{k}$ C21 results from ref. ${ }^{15 \mathrm{a}}$ and additional results for hexamethylenetetramine and succinic acid from refs. ${ }^{34}$ Differences for oxalic acid are large (between -15.8 and $-26.8 \mathrm{~kJ}$ mol-1) and appear not to account for the rearrangement energy from crystal to gas phase.

1 Unit cells kept at experimental values and atomic positions fully optimized; geometric counterpoise correction..$^{35}$

m Unit cell and atomic positions fully optimized; counterpoise correction implicitly included in $\mathrm{HF}-3 \mathrm{c}$ method. . $^{15}$

n Single point energies at B3LYP-D*/TZP optimized geometries; BSSE corrected. . $^{\text {sc }}$

o Intermolecular force fields used to optimize unit cells, atomic positions and compute lattice energies..$^{36}$

p Unit cell and atomic positions fully optimized..$^{15 b}$

q Unit cell and atomic positions fully optimized. ${ }^{33}$ 
3.2. G60 and K7 comparisons. The performance of the CE-B3LYP model for the G60 and K7 sets of crystals is summarized in Table 2, where comparisons can be made with B3LYPD*/TZP (K7 only), SP-B3LYP-D*, HF-3c and S-HF-3c results, , ${ }^{\text {isc }}$ as well as B3LYP-D*/631G(d,p) and PIXEL results. ${ }^{17}$ Figure 2 plots deviations from benchmark lattice energies for all of these computational approaches, and for all G60 crystals. Note that the G60 and K7 benchmark energies derive from experimental sublimation enthalpies back-corrected by $\sim 5 \mathrm{~kJ} \mathrm{~mol}^{-1}(=2 R T$ for polyatomics), and the results from periodic crystal minus molecule approaches implicitly take into account a relaxation energy term but, in contrast to the X23 set, this correction has not been made to any CE-B3LYP and PIXEL lattice energies for these sets of crystals. For the G60 set the CE-B3LYP model performs remarkably well overall and, perhaps surprisingly, better than all other methods in Table 2, with a small MD and a MAD of only $8.4 \mathrm{~kJ} \mathrm{~mol}^{-1}$. This MAD for the G60 set is greater than for the X23 set, and slightly larger than expected from the ratio of average lattice energies for the two sets. As observed for the X23 set, this performance is of similar quality to the two B3LYP plus dispersion approaches. Statistics for the K7 set in Table 2 echo the conclusions from the G60 set, but with smaller values of MAD, MD and SD that reflect the much smaller average lattice energy for this set.

Deviations from benchmark experimental lattice energies for the G60 set (Figure 2) reveal several trends and systematic anomalies. For example, it is clear from the plot that the HF-3c model systematically overbinds many crystals, and although the dispersion-scaled variant S-HF$3 \mathrm{c}$ is an improvement, this refinement tends to do little more overall than displace the HF-3c trendline by $\sim 10-15 \mathrm{~kJ} \mathrm{~mol}^{-1}$. For most of the crystals the CE-B3LYP trendline closely follows those for the B3LYP-D* and SP-B3LYP-D* results. Bearing in mind the use of many different geometries, several models display overbinding for crystals of melamine (MELAMI04), oxalic acid (OXALAC04), urea (UREAXX09) and N,N-dimethylurea (WIFKEB), and we attribute this to the lack (or inadequacy) of a relaxation energy correction.

3.3. Comparison between CE-B3LYP and PIXEL lattice energies. The full set of G60 lattice energies obtained by CE-B3LYP and PIXEL approaches, with identical crystal 
structures used for both calculations (see Table S2), provides a unique opportunity to make a quantitative comparison between the two models, and explore how similar - or different - are the individual terms in eq. (2). This sort of comparison has so far been limited to energies for small numbers of molecular pairs; ${ }^{; a}$ here we have sums over large numbers of molecular pairs, at a wide range of mutual orientations and separations, for each of the molecular crystals in the G60 set. A simple regression analysis reveals that overall $E_{\text {lat }}^{\text {CE-B3LYP }}=1.062 E_{\text {lat }}^{\text {PIXEL }}$, but the differences between the two can be as large as $+30.7 \mathrm{~kJ}^{-1}$ (DIMNAN01) and $-25.2 \mathrm{~kJ} \mathrm{~mol}^{-1}$ (HCLBNZ11). The MD of $5.9 \mathrm{~kJ} \mathrm{~mol}^{-1}$ follows from the values in Table 2, and the MAD between the two sets of results is $9.8 \mathrm{~kJ} \mathrm{~mol}^{-1}$. From this we can conclude that CE-B3LYP lattice energies are on average $\sim 6 \%$ larger than those from PIXEL, but there can be substantial differences between the two.

To explore further the relationship between CE-B3LYP and PIXEL energies, Figure 3 plots individual components of CE-B3LYP lattice energies against the respective PIXEL energies for the G60 crystals (i.e., the lattice sums of electrostatic, polarization, dispersion and repulsion energies for the two models). Before discussing these plots it is important to recognize that the CE-B3LYP energy terms in Figure 3 are unscaled - the primed terms in eq. (3). It is readily seen from the figure that electrostatic, dispersion and repulsion energy terms are remarkably close for these two models. This is not surprising for the electrostatic energy (even though CE-B3LYP uses B3LYP/6-31G(d,p) monomer electron densities, and the PIXEL results are based on MP2/6$31 \mathrm{G}(\mathrm{d}, \mathrm{p})$ electron densities), but the close similarity of dispersion and repulsion energy terms for the two models is surprising. Given the very different ways in which these two terms have been derived in the two models, the close similarity of these energies in Figure 3 strongly supports an argument that they both represent realistic models of those phenomena. There are, however, some large differences between CE-B3LYP and PIXEL polarization energies. Although the majority of these energies for the G60 set are smaller in magnitude than $20 \mathrm{~kJ} \mathrm{~mol}^{-1}$, PIXEL polarization energies are typically more negative than those in the CE-B3LYP model, and often much more negative. It is tempting to attribute the largest differences between the two lattice 
energies to this term, but that is not the case; there is in fact no single factor responsible for these differences. For dimethylnitroaniline (DIMNAN01) separate CE-B3LYP and PIXEL lattice energy components agree quite well, but the CE-B3LYP scale factors (eq. (3)) lead to a substantial difference between the two lattice energies: $E_{\text {lat }}^{\text {CE-B3LYP }}=-121.7 \mathrm{~kJ} \mathrm{~mol}^{-1}$ and $E_{\text {lat }}^{\mathrm{PIXEL}}=$ $-91.0 \mathrm{~kJ} \mathrm{~mol}{ }^{-1}$, results that bracket the benchmark value of $-107.7 \mathrm{~kJ} \mathrm{~mol}^{-1}$. The situation for hexachlorobenzene (HCLBNZ11) is different; the sum of PIXEL polarization and dispersion energy terms is much more negative than from CE-B3LYP, and by nearly $50 \mathrm{~kJ} \mathrm{~mol}^{-1}$, with the result that $E_{\text {lat }}^{\mathrm{CE}-\mathrm{B} 3 \mathrm{LYP}}=-68.3 \mathrm{~kJ} \mathrm{~mol}^{-1}$ and $E_{\text {lat }}^{\mathrm{PIXL}}=-93.5 \mathrm{~kJ} \mathrm{~mol}^{-1}$, the latter being much closer to the benchmark value of $-93.3 \mathrm{~kJ} \mathrm{~mol}^{-1}$, which is associated with considerable uncertainty (see footnote to Supporting Information Table S2).

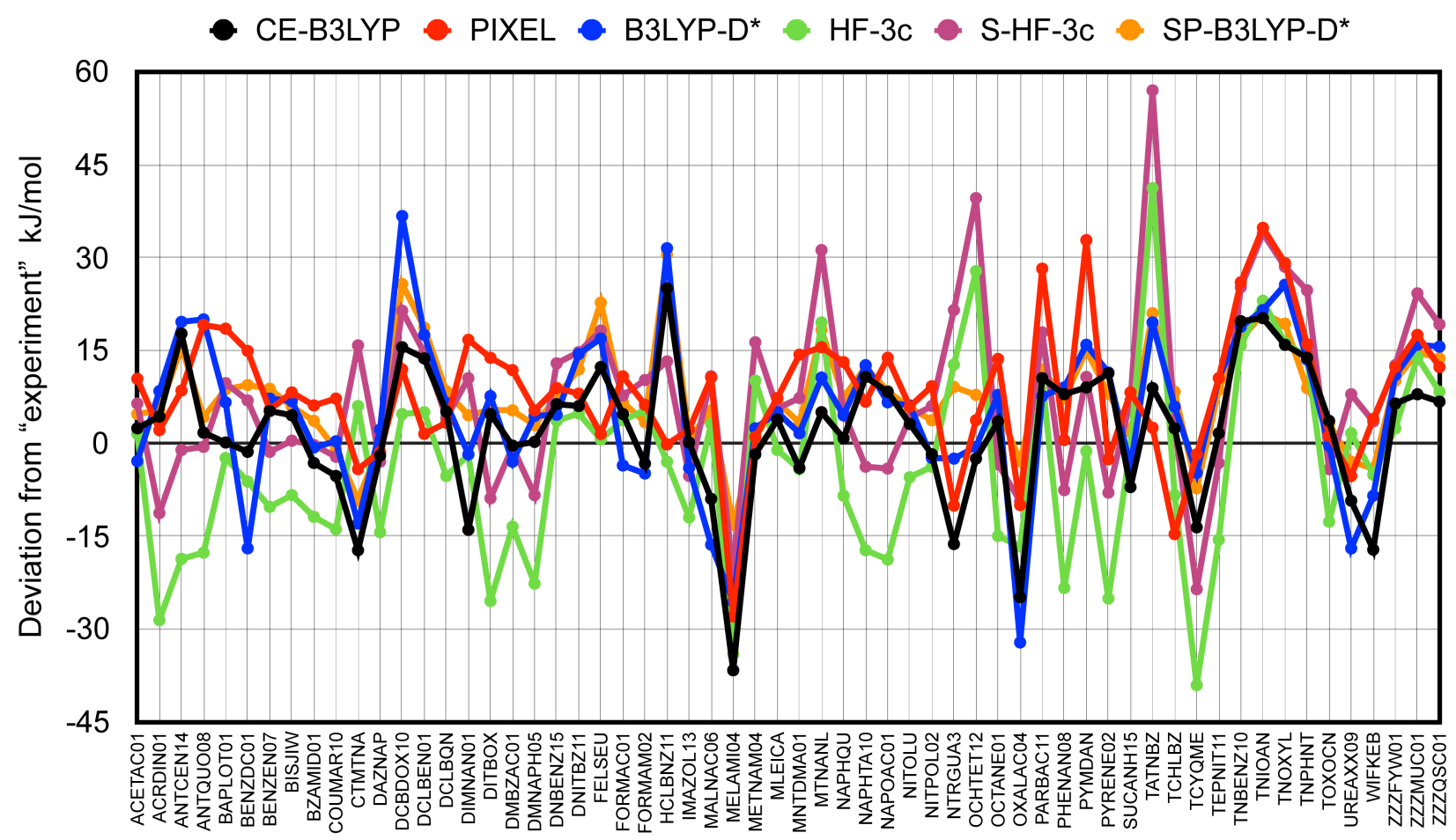

Figure 2. Deviation of computational estimates of lattice energies from the G60 benchmarks. Note that the vertical axis spans more than twice the range of that in Figure 1. 
Table 2. Mean absolute deviation (MAD), mean signed deviation (MD), standard deviation from the mean (SD), all in $\mathrm{kJ} \mathrm{mol}^{-1}$, and mean absolute percent deviation (MA\%D) of computational estimates of lattice energies from the G60 and K7 benchmarks.

\begin{tabular}{|l|l|c|c|c|c|}
\hline & & MAD & MD & SD & MA\%D \\
\hline G60 set & & & & & \\
\hline & CE-B3LYP & 8.4 & 2.0 & 10.9 & 8.0 \\
\hline & SP-B3LYP-D*。 & 9.1 & 7.7 & 8.1 & 8.8 \\
\hline & B3LYP-D*/6-31G(d,p) & 10.6 & 5.1 & 12.5 & 10.2 \\
\hline & PIXEL ${ }^{\alpha}$ & 10.5 & 7.9 & 11.1 & 10.7 \\
\hline & S-HF-3c ${ }^{d}$ & 12.0 & 7.6 & 14.2 & 10.8 \\
\hline & HF-3c ${ }^{d}$ & 12.1 & -3.6 & 15.0 & 11.7 \\
\hline & & & & & \\
\hline & B3LYP-D*/TZP & 4.0 & 0.8 & 4.7 & 10.7 \\
\hline & SP-B3LYP-D* & 4.1 & 1.5 & 4.3 & 11.1 \\
\hline & CE-B3LYP & 4.6 & -2.7 & 5.6 & 8.3 \\
\hline & S-HF-3c ${ }^{d}$ & 6.2 & -3.7 & 8.5 & 9.7 \\
\hline & HF-3c ${ }^{d}$ & 8.1 & -8.1 & 9.2 & 15.0 \\
\hline
\end{tabular}

a Present work; crystal structures from ref. " for the G60 set and from ref. is for the K7 set (for details see Tables S2 and S3).

b SP-B3LYP-D* denotes single point B3LYP-D*/TZP energies using optimized crystal geometries computed with a dispersion-scaled HF-3c-(0.27 s8) method..$^{\text {ss }}$

c Experimental crystal structures."

d S-HF-3c is also denoted HF-3c-(0.7 s8), and is a dispersion-scaled variant of HF-3c; unit cell and atomic positions fully optimized. ${ }^{\text {sse }}$

e Unit cell and atomic positions fully optimized.st 

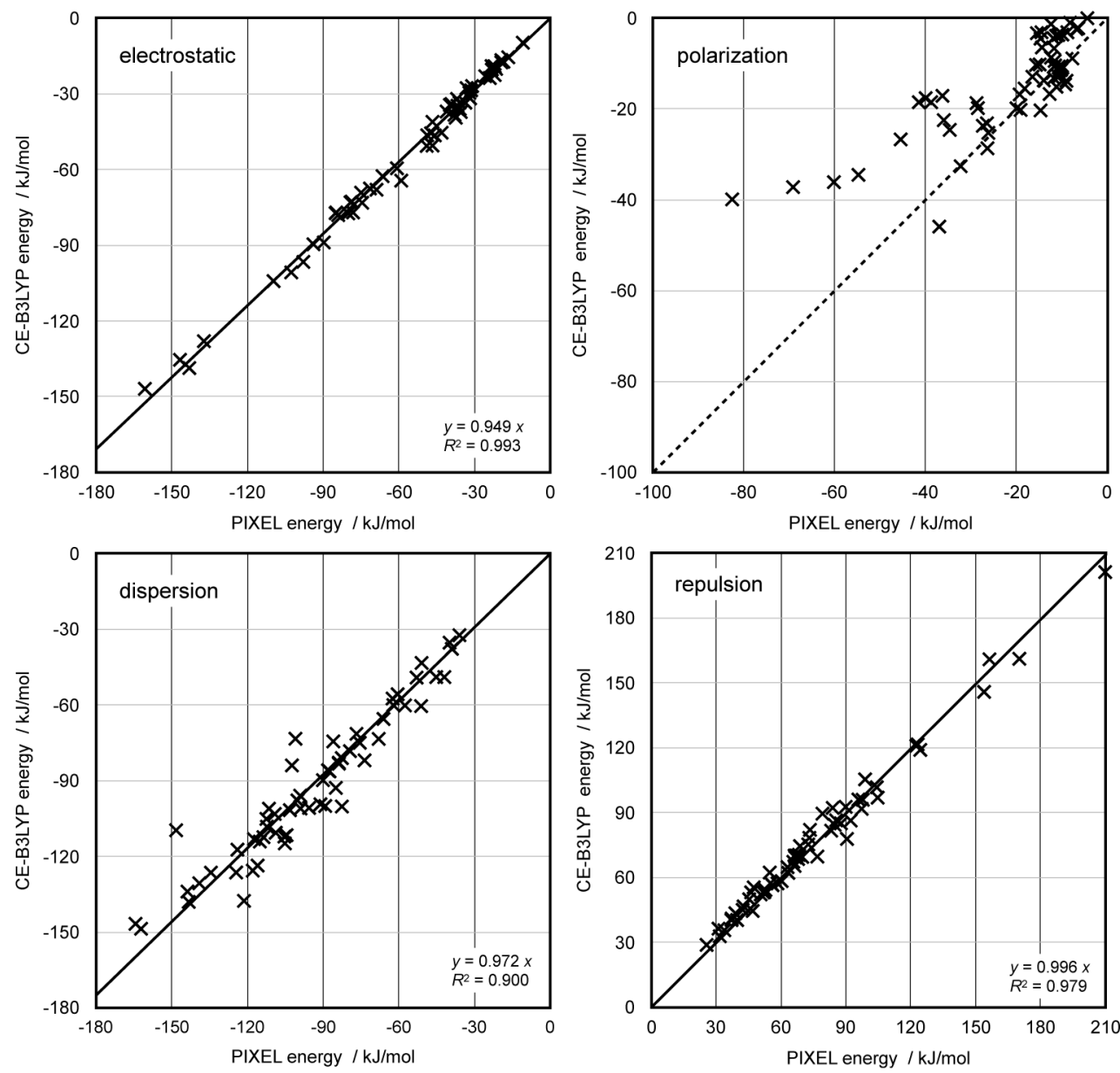

Figure 3. Comparison between individual CE-B3LYP and PIXEL lattice energy components for crystals in the G60 set. Although all plots are the same size, note that the energy scales are not all the same, and in particular the scale for the polarization energy is roughly half that for the other terms. For electrostatic, dispersion and repulsion components the line of best fit through the origin is displayed, with the equation at bottom right, along with the $R^{2}$ statistic. For the polarization energy the dashed line is simply a line of unit slope through the origin. 
Table 3. Mean absolute deviation (MAD), mean signed deviation (MD), standard deviation from the mean (SD), all in $\mathrm{kJ} \mathrm{mol}^{-1}$, and mean absolute percent deviation (MA\%D) of computational estimates of lattice energies from the Z20 benchmarks.

\begin{tabular}{|l|l|c|c|c|c|}
\hline & & MAD & MD & SD & MA\%D \\
\hline Z20 set & & & & & \\
\hline & CE-B3LYP & 2.8 & 0.2 & 3.9 & 9.2 \\
\hline & CCSD(T)/CBS fragments ${ }^{\circ}$ & 4.8 & 4.5 & 6.1 & 12.7 \\
\hline
\end{tabular}

a Present work; based on the same set of experimental crystal structures used in ref. ${ }^{\text {san }}$ (see Table S4).

b CCSD(T)/CBS fragment-based approach, using experimental crystal structures ${ }^{132}$ (Table S4).

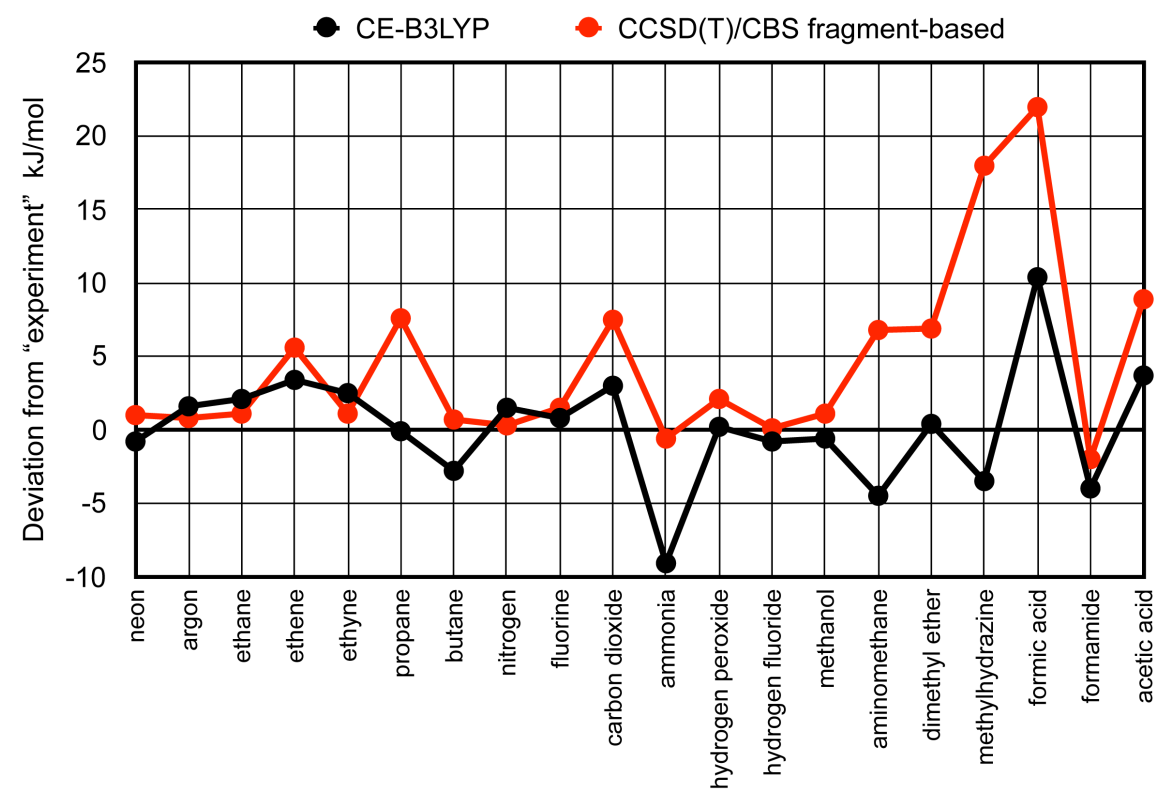

Figure 4. Deviation of computational estimates of lattice energies from the Z20 benchmarks. 
3.4. Z20 comparisons. This set of crystal structures includes two noble gases, three diatomics, small hydrocarbons and organics, and as a consequence the average benchmark lattice energy of $-41.7 \mathrm{~kJ} \mathrm{~mol}^{-1}$ is the lowest of the benchmark sets considered here. As described earlier, the lattice energies used as benchmarks here are based on experimental sublimation enthalpies carefully adjusted to $0 \mathrm{~K}$, and corrected for computed values of relaxation and zeropoint energies. Table 3 and Figure 4 compare the present CE-B3LYP results (see also Table S4) with those obtained from an additive scheme based on $\operatorname{CCSD}(\mathrm{T})$ energies of two-, three- and four-body interactions, with estimated extrapolation to the complete basis set (CBS) limit. ${ }^{13 a}$ Both computational estimates of lattice energies are based on the same experimental crystal structures, enabling a very direct comparison between the two sets of results. Perhaps surprisingly, given the relative computational cost of the two approaches, the statistics in Table 4 show that overall the CE-B3LYP model provides better estimates of lattice energies for these crystals, and Figure 4 identifies the main reason: the $\operatorname{CCSD}(\mathrm{T}) / \mathrm{CBS}$ fragment-based method significantly underbinds the organic molecular crystals aminomethane, dimethyl ether, methylhydrazine, formic acid and acetic acid. Červinka et al., ${ }^{\text {sa }}$ identified several ways in which their fragment-based model could be improved, and concluded that the overall uncertainty of their $\operatorname{CCSD}(\mathrm{T}) / \mathrm{CBS}$ lattice energy estimates amounted to $10-20 \%$.

3.5. Lattice energies for polymorphs and large molecules. In our recent work ${ }^{s_{a}}$ we were somewhat pessimistic about the ability of CE-B3LYP or PIXEL model energies to provide reliable estimates of lattice energy differences between polymorphs. This was based on the conclusion by Nyman and Day" that "polymorphic lattice energy differences are typically very small: over half of polymorph pairs are separated by less than $2 \mathrm{~kJ} \mathrm{~mol}^{-1}$ and lattice energy differences exceed $7.2 \mathrm{~kJ} \mathrm{~mol}^{-1}$ in only $5 \%$ of cases." Consideration of the MAD, SD and MA\%D statistics obtained for benchmark crystals above might suggest that our pessimism was justified. But those statistics refer to deviations from benchmark values and, although those values are the best presently available, there can be no question that they have considerable uncertainty associated with them. Computing lattice energy differences between polymorphs avoids some of 
the problems inherent in comparisons with benchmark values, namely the unavoidable uncertainty in $\Delta H_{\text {sub }}(T)$ (estimated to be $\sim 5 \mathrm{~kJ} \mathrm{~mol}^{-1}$ ) and, for rigid molecules, the need to consider the relaxation energy, $\Delta E_{\text {relax }}$, in eq. (4). Two examples, 1,4-diiodobenzene and 5fluorouracil, provide evidence that CE-B3LYP model energies may in fact be capable of providing useful estimates of lattice energy differences in these circumstances.

1,4-diiodobenzene is known to have two forms: the $\alpha$-form is stable at room temperature, transforming into a high-temperature $\beta$-form at $\sim 326 \mathrm{~K} .{ }^{38}$ CE-B3LYP lattice energies, using B3LYP/DGDZVP electron distributions, based on two crystal structure determinations of the $\alpha$ form (ZZZPRO03, ZZZPRO06) are -86.5 and $-87.3 \mathrm{~kJ} \mathrm{~mol}^{-1}\left(\right.$ mean $\left.=-86.9 \mathrm{~kJ} \mathrm{~mol}^{-1}\right)$. For two $\beta$ form structures (ZZZPRO04, ZZZPRO07) the lattice energies are -84.9 and $-85.3 \mathrm{~kJ} \mathrm{~mol}^{-1}$ (mean $\left.=-85.1 \mathrm{~kJ} \mathrm{~mol}^{-1}\right)$. From these we conclude that a conservative estimate of the lattice energy difference between the two forms is $-2 \pm 1 \mathrm{~kJ} \mathrm{~mol}^{-1}$, with the $\alpha$-form lower in energy. This very small difference is in excellent agreement with the conclusions from a computationally-intensive diffusion Monte Carlo study, ${ }^{39}$ where the difference was estimated to be between $-2 \pm 1$ and $-8 \pm$ $3 \mathrm{kcal} / \mathrm{mol} / \mathrm{cell}=-2$ to $-8 \mathrm{~kJ} \mathrm{~mol}^{-1}$, based on the experimental crystal structures ZZZPRO03 and ZZZPRO04. (For reference, the most recent measurement of the sublimation enthalpy of 1,4diiodobenzene is $85.4 \pm 0.4 \mathrm{~kJ} \mathrm{~mol}^{-1}$, corrected to room temperature. ${ }^{40}$ )

Two polymorphs of 5-fluorouracil are known: the original form I, with four molecules in the asymmetric unit, and form II with one molecule, the latter discovered as the result of an extensive computational crystal structure prediction study.4 We have computed CE-B3LYP lattice energies for the two forms based on crystal structures at room-temperature and at $150 \mathrm{~K}$. At RT the lattice energies of form I (FURACL; -124.4 $\mathrm{kJ} \mathrm{mol}^{-1}$ ) and form II (FURACL02; $135.4 \mathrm{~kJ} \mathrm{~mol}^{-1}$ ) indicate that form II is more stable by $-11.0 \mathrm{~kJ} \mathrm{~mol}^{-1}$, while at $150 \mathrm{~K}$ the lattice energies of form I (FURACL01; -133.5 kJ mol-1) and form II (FURACL03; -137.2 $\mathrm{kJ} \mathrm{mol}^{-1}$ ) indicate that form II is more stable by $-3.7 \mathrm{~kJ} \mathrm{~mol}^{-1}$. These results are in excellent agreement with 
the original computational study, ${ }^{41}$ which obtained lattice energy differences of $-9.9 \mathrm{~kJ}^{\mathrm{mol}}{ }^{-1}$ using RT structures and $-6.3 \mathrm{~kJ} \mathrm{~mol}^{-1}$ for $150 \mathrm{~K}$ structures. (The most recent measurement of the sublimation enthalpy of 5-fluorouracil is $132.5 \pm 1.2 \mathrm{~kJ} \mathrm{~mol}^{-1}$, corrected to room temperature. ${ }^{42}$ )

The calculation of CE-B3LYP lattice energies using CrystalExplorer17 ${ }^{23}$ is straightforward, and because the computation of individual pairwise model energies is much faster than actual DFT calculations, the approach can be readily applied to crystal structures of quite large molecules. For coronene, $\mathrm{C}_{24} \mathrm{H}_{12}$, the CE-B3LYP lattice energy is $-134.9 \mathrm{~kJ} \mathrm{~mol}^{-1}$ based on the RT crystal structure CORONE. This result may be compared with theoretical estimates obtained using periodic boundary conditions as well as pairwise sums of energies (also using the CORONE crystal structure), which yielded a range of values between -133 and $-172 \mathrm{~kJ} \mathrm{~mol}^{-1} .^{43}$ The recommended value of $\Delta H_{\text {sub }}(R T)$ is $142.6 \pm 8.7 \mathrm{~kJ} \mathrm{~mol}^{-1,4}$ and the relaxation energy for coronene is expected to be negligible. For the much larger hydrocarbon rubrene, $\mathrm{C}_{42} \mathrm{H}_{28}$, the CEB3LYP lattice energy is $-202.2 \mathrm{~kJ} \mathrm{~mol}^{-1}$ based on the RT crystal structure QQQCIG11. The recommended (and only) value of $\Delta H_{\text {sub }}(R T)$ is $180.6 \mathrm{~kJ} \mathrm{~mol}^{-1}$, which was accompanied by the comment "questionable value" ${ }^{44}$ For rubrene the relaxation energy is likely to be substantial, so we would expect this CE-B3LYP lattice energy to overestimate the sublimation enthalpy.

\section{CONCLUSIONS}

We undertook the calculations reported here in order to clearly establish the level of confidence for lattice energies estimated using the CE-B3LYP model energies. From the results across four different benchmark sets of (largely organic) molecular crystals we conclude that CE-B3LYP lattice energies - based on experimental crystal structures - compare very well with available experimental benchmark estimates derived from sublimation enthalpies, and in many cases they are comparable with (and sometimes better than) the results of more computationally-demanding approaches based on periodic DFT plus dispersion methodologies. From the statistics in Tables 1 to 4 the MAD of CE-B3LYP results from experimental benchmark values is in the approximate 
range 3 to $8 \mathrm{~kJ} \mathrm{~mol}^{-1}$, with the actual values dependent on the magnitude of energies in each set. Mean absolute percentage deviations are in the narrower range of 7 to $9 \%$.

Perhaps a better way of assessing the overall performance of the CE-B3LYP lattice energies is to examine statistics for its performance over the complete set of 110 crystals (i.e., all of X23, $\mathrm{G} 60, \mathrm{~K} 7$ and $\mathrm{Z20}$ ): $\mathrm{MD}=0.8 \mathrm{~kJ} \mathrm{~mol}^{-1}, \mathrm{MAD}=6.6 \mathrm{~kJ} \mathrm{~mol}^{-1}, \mathrm{SD}$ from the mean $=9.1 \mathrm{~kJ} \mathrm{~mol}^{-1}$ and $\mathrm{MA} \% \mathrm{D}=8.2 \%$. We believe these figures provide the best available estimate of the level of confidence that may be placed on CE-B3LYP lattice energies computed with experimental crystal structure geometries. However, it is important to bear in mind that these statistics refer to comparisons with experimental benchmark lattice energies, and that there is reason to be more optimistic when comparing computed lattice energies for polymorphs, especially for rigid molecules. Although the the present methodology lacks a systematic way of estimating the very important relaxation energy for molecules with conformational freedom, in separate work we have described a successful computational approach to this problem, and demonstrated its application to the seven polymorphs of 5-methyl-2-[(2-nitrophenyl)amino]-3thiophenecarbonitrile (ROY).45

The results described in this work highlight the need for particular care when interpreting differences in performance between various computational methods, especially the need to appreciate the consequences of using different sets of crystal/molecule geometries. Even where computational approaches make use of experimental crystal structures it is not clear that authors are aware of the dependence of results on the temperature of the structure determination (i.e., the particular crystal structures chosen), or the need to normalize bond lengths to $\mathrm{H}$ atoms, as routinely performed in CE-B3LYP and PIXEL calculations. There are many instances in the literature where crystal structures based on X-ray diffraction data have been used uncritically, and "without further modification". In Section 2.1 we noted that the combined set of 110 lattice energies includes three pairs of duplicate structures, some measured at different temperatures. For the two acetic acid structures, ACETAC01 (278 K) and ACETAC05 (4 K), CE-B3LYP lattice energies are -70.4 and $-75.5 \mathrm{~kJ} \mathrm{~mol}^{-1}$, respectively. For benzene, BENZEN (218 K) and 
BENZEN07 (123 K), CE-B3LYP lattice energies are -47.3 and $-50.1 \mathrm{~kJ} \mathrm{~mol}^{-1}$, respectively. And for urea, where both structures UREAXX and UREAXX09 refer to room temperature, CEB3LYP lattice energies are -104.2 and $-111.8 \mathrm{~kJ} \mathrm{~mol}^{-1}$, respectively. From this the dependence on temperature can be seen to be as much as $10 \%$, but there are also systematic errors that may be present in any particular crystal structure determination.

This work has also identified a need for more extensive benchmark sets of crystal structures and associated 'experimental' lattice energies. In particular, we emphasize the absence in the current benchmark sets of organic molecules containing atoms such as B, F, P, S, As, Se, Br and I. Compiling a database incorporating molecules containing atoms such as these will require a careful assessment of the available sublimation enthalpy (and possibly other phase-change) data from the recent compilations of Chickos and Acree, ${ }^{10}$ preferably for molecules that are essentially rigid, and cross-referencing with the availability of crystal structures. Although we have already looked into this, we concluded that this will be better undertaken independently of the present work; it is not a trivial exercise. The present work has made it very clear that the most valuable benchmark data are those that have been most critically assessed. 


\section{ASSOCIATED CONTENT}

\section{Supporting Information.}

The following files are available free of charge. Tables of CE-B3LYP lattice energies and benchmark lattice energies used in the statistical analyses for all molecular crystals in the X23, G60, K7 and Z20 benchmark sets (Tables S1 to S4) and a table of cell dipole energies for polar space groups (Table S5). (PDF)

\section{AUTHOR INFORMATION}

\section{Corresponding Author}

*E-mail: mark.spackman@uwa.edu.au

\section{Present Addresses}

$\S$ Center for Materials Crystallography, Department of Chemistry, Aarhus University, DK-8000

Aarhus C, Denmark

\section{ACKNOWLEDGMENTS}

This work has been supported by the Australian Research Council (DP130103304) and the Danish National Research Foundation (Center for Materials Crystallography, DNRF-93).

\section{REFERENCES}

(1) (a) Hermann, J.; DiStasio, R. A.; Tkatchenko, A. First-Principles Models for van der Waals Interactions in Molecules and Materials: Concepts, Theory, and Applications. Chem. Rev. 2017, 117, 4714-4758. (b) Hoja, J.; Reilly, A. M.; Tkatchenko, A. First-principles modeling of molecular crystals: structures and stabilities, temperature and pressure. WIREs Comput. Mol. Sci. 2017, 7, e1294. (c) Beran, G. J. Modeling Polymorphic Molecular Crystals with Electronic Structure Theory. Chem. Rev. 2016, 116, 5567-5613. (d) Grimme, S.; Hansen, A.; Brandenburg, J. G.; Bannwarth, C. Dispersion-Corrected Mean-Field Electronic Structure Methods. Chem. Rev. 2016, 116, 5105-5154.

(2) Spackman, M. A.; Jayatilaka, D. Hirshfeld Surface Analysis. CrystEngComm 2009, 11, 19-32. 
(3) Turner, M. J.; McKinnon, J. J.; Jayatilaka, D.; Spackman, M. A. Visualisation and characterisation of voids in crystalline materials. CrystEngComm 2011, 13, 1804-1813.

(4) (a) Spackman, M. A.; McKinnon, J. J.; Jayatilaka, D. Electrostatic Potentials Mapped on Hirshfeld Surfaces Provide Direct Insight into Intermolecular Interactions in Crystals. CrystEngComm 2008, 10, 377-388. (b) Edwards, A. J.; Mackenzie, C. F.; Spackman, P. R.; Jayatilaka, D.; Spackman, M. A. Intermolecular interactions in molecular crystals: What's in a name? Faraday Discuss. 2017, 203, 93-112.

(5) (a) Mackenzie, C. F.; Spackman, P. R.; Jayatilaka, D.; Spackman, M. A. CrystalExplorer model energies and energy frameworks: Extension to metal coordination compounds, organic salts, solvates and open shell systems. IUCrJ 2017, 4, 575-587. (b) Turner, M. J.; Grabowsky, S.; Jayatilaka, D.; Spackman, M. A. Accurate and efficient model energies for exploring intermolecular interactions in molecular crystals. J. Phys. Chem. Lett. 2014, 5, 4249-4255.

(6) Turner, M. J.; Thomas, S. P.; Shi, M. W.; Jayatilaka, D.; Spackman, M. A. Energy frameworks: insights into interaction anisotropy and the mechanical properties of molecular crystals. Chem. Commun. 2015, 51, 3735-3738.

(7) (a) Dey, D.; Bhandary, S.; Thomas, S. P.; Spackman, M. A.; Chopra, D. Energy frameworks and a topological analysis of the supramolecular features in in situ cryocrystallized liquids: tuning the weak interaction landscape via fluorination. Phys. Chem. Chem. Phys. 2016, 18, 31811-31820. (b) Dey, D.; Thomas, S. P.; Spackman, M. A.; Chopra, D. 'Quasi-isostructural polymorphism' in molecular crystals: inputs from interaction hierarchy and energy frameworks. Chem. Commun. 2016, 52, 2141-2144.

(8) Thomas, S. P.; Shi, M. W.; Koutsantonis, G. A.; Jayatilaka, D.; Edwards, A. J.; Spackman, M. A. The elusive structural origin of plastic bending in dimethyl sulfone crystals with quasiisotropic crystal packing. Angew. Chem. Int. Ed. 2017, 56, 8468-8472. 
(9) (a) Eikeland, E.; Spackman, M. A.; Iversen, B. B. Quantifying Host-Guest Interaction Energies in Clathrates of Dianin's Compound. Cryst. Growth Des. 2016, 16, 6858-6866. (b) Eikeland, E.; Thomsen, M. K.; Madsen, S. R.; Overgaard, J.; Spackman, M. A.; Iversen, B. B. Structural Collapse of the Hydroquinone-Formic Acid Clathrate: A Pressure-Medium-Dependent Phase Transition. Chem. Eur. J. 2016, 22, 4061-4069. (c) Eikeland, E.; Thomsen, M. K.; Overgaard, J.; Spackman, M. A.; Iversen, B. B. Intermolecular Interaction Energies in Hydroquinone Clathrates at High Pressure. Cryst. Growth Des. 2017, 17, 3834-3846.

(10) (a) Acree, W.; Chickos, J. S. Phase Transition Enthalpy Measurements of Organic and Organometallic Compounds. Sublimation, Vaporization and Fusion Enthalpies From 1880 to 2015. Part 1. C1 - C10. J. Phys. Chem. Ref. Data 2016, 45, 033101. (b) Acree, W.; Chickos, J. S. Phase Transition Enthalpy Measurements of Organic and Organometallic Compounds and Ionic Liquids. Sublimation, Vaporization, and Fusion Enthalpies from 1880 to 2015. Part 2. C11-C192. J. Phys. Chem. Ref. Data 2017, 46, 013104.

(11) (a) Gavezzotti, A. Non-conventional Bonding between Organic Molecules. The 'Halogen Bond' in Crystalline Systems. Mol. Phys. 2008, 106, 1473-1485. (b) Gavezzotti, A. Calculation of lattice energies of organic crystals: the PIXEL integration method in comparison with more traditional methods. Z. Kristallogr. 2005, 220, 499-510. (c) Gavezzotti, A. Calculation of Intermolecular Interaction Energies by Direct Numerical Integration over Electron Densities. 2. An Improved Polarization Model and the Evaluation of Dispersion and Repulsion Energies. $J$. Phys. Chem. B 2003, 107, 2344-2353. (d) Gavezzotti, A. Calculation of Intermolecular Interaction Energies by Direct Numerical Integration over Electron Densities. 1. Electrostatic and Polarization Energies in Molecular Crystals. J. Phys. Chem. B 2002, 106, 4145-4154.

(12) Allen, F. H.; Watson, D. G.; Brammer, L.; Orpen, A. G.; Taylor, R. In International Tables for Crystallography; 3rd ed.; Prince, E., Ed.; Springer Verlag: Berlin, 2004; Vol. C: Mathematical, physical and chemical tables, p 790-811. 
(13) (a) Červinka, C.; Fulem, M.; Růžička, K. CCSD(T)/CBS fragment-based calculations of lattice energy of molecular crystals. J. Chem. Phys. 2016, 144, 064505. (b) Červinka, C.; Fulem, M. State-of-the-Art Calculations of Sublimation Enthalpies for Selected Molecular Crystals and Their Computational Uncertainty. J. Chem. Theory Comput. 2017, 13, 2840-2850.

(14) Chickos, J. S. Enthalpies of sublimation after a century of measurement: A view as seen through the eyes of a collector. Netsu Sokutei 2003, 39, 116-124.

(15) (a) Otero de la Roza, A.; Johnson, E. J. A Benchmark for Non-Covalent Interactions in Solids. J. Chem. Phys. 2012, 137, 054103. (b) Reilly, A. M.; Tkatchenko, A. Understanding the role of vibrations, exact exchange, and many-body van der Waals interactions in the cohesive properties of molecular crystals. J. Chem. Phys. 2013, 139, 024705. (c) Cutini, M.; Civalleri, B.; Corno, M.; Orlando, R.; Brandenburg, J. G.; Maschio, L.; Ugliengo, P. Assessment of Different Quantum Mechanical Methods for the Prediction of Structure and Cohesive Energy of Molecular Crystals. J. Chem. Theory Comput. 2016, 12, 3340-3352.

(16) (a) Gavezzotti, A.; Filippini, G. In Theoretical aspects and computer modeling of the molecular solid state; Gavezzotti, A., Ed.; Wiley: Chichester, 1997, p 61-97. (b) Buchholz, H. K.; Hylton, R. K.; Brandenburg, J. G.; Seidel-Morgenstern, A.; Lorenz, H.; Stein, M.; Price, S. L. Thermochemistry of Racemic and Enantiopure Organic Crystals for Predicting Enantiomer Separation. Cryst. Growth Des. 2017, 17, 4676-4686.

(17) Maschio, L.; Civalleri, B.; Ugliengo, P.; Gavezzotti, A. Intermolecular Interaction Energies in Molecular Crystals: Comparison and Agreement of Localized Møller-Plesset 2, Dispersion-corrected Density Functional, and Classical Empirical Two-body Calculations. $J$. Phys. Chem. A 2011, 115, 11179-11186.

(18) (a) Kitaura, K.; Morokuma, K. New Energy Decomposition Scheme for Molecular Interactions within Hartree-Fock Approximation. Int. J. Quantum Chem. 1976, 10, 325-340. (b) Ziegler, T.; Rauk, A. CO, CS, N2, PF3, and $\mathrm{CNCH} 3$ as sigma donors and pi acceptors - 
Theoretical study by the Hartree-Fock-Slater transition state method. Inorg. Chem. 1979, 18, 1755-1759.

(19) (a) Hayes, I. C.; Stone, A. J. An Intermolecular Perturbation-Theory for the Region of Moderate Overlap. Mol. Phys. 1984, 53, 83-105. (b) Jeziorski, B.; Moszynski, R.; Szalewicz, K. Perturbation Theory Approach to Intermolecular Potential Energy Surfaces of van der Waals Complexes. Chem. Rev. 1994, 94, 1887-1930.

(20) Su, P.; Li, H. Energy Decomposition Analysis of Covalent Bonds and Intermolecular Interactions. J. Chem. Phys. 2009, 131, 014102.

(21) Thakkar, A. J.; Lupinetti, C. In Atoms, Molecules and Clusters in Electric Fields; Maroulis, G., Ed.; World Scientific: 2006, p 505-529.

(22) Grimme, S. Semiempirical GGA-Type Density Functional Constructed with a LongRange Dispersion Correction. J. Comput. Chem. 2006, 27, 1787-1799.

(23) Turner, M. J.; McKinnon, J. J.; Wolff, S. K.; Grimwood, D. J.; Spackman, P. R.; Jayatilaka, D.; Spackman, M. A., CrystalExplorer17, University of Western Australia. http://hirshfeldsurface.net, 2017.

(24) Spackman, P. R. PhD thesis, University of Western Australia, 2017.

(25) (a) Dunitz, J. D.; Gavezzotti, A. Proteogenic amino acids: chiral and racemic crystal packings and stabilities. J. Phys. Chem. B 2012, 116, 6740-6750. (b) Abramov, Y. A. Understanding the Risk of Agglomeration of Polar Pharmaceutical Crystals. Cryst. Growth Des. 2017, 17, 2873-2880. (c) van Eijck, B. P.; Kroon, J. Coulomb energy of polar crystals. J. Phys. Chem. B 1997, 101, 1096-1100.

(26) Godfrey, P. D.; Mirabella, M. J.; Brown, R. D. Structural Studies of Higher Energy Conformers by Millimeter-Wave Spectroscopy: Oxalic Acid. J. Phys. Chem. A 2000, 104, 258264. 
(27) (a) Rousseau, B.; Van Alsenoy, C.; Keuleers, R.; Dessyn, H. O. Solids Modeled by AbInitio Crystal Field Methods. Part 17. Study of the Structure and Vibrational Spectrum of Urea in the Gas Phase and in Its P421m Crystal Phase. J. Phys. Chem. A 1998, 102, 6540-6548. (b) Brown, R. D.; Godfrey, P. D.; Storey, J. The microwave spectrum of urea. J. Mol. Spec. 1975, 58, 445-450. (c) Godfrey, P. D.; Brown, R. D.; Hunter, A. N. The shape of urea. J. Mol. Struct. $1997,413-414,405-414$.

(28) Alonso, J. L.; Vaquero, V.; Pena, I.; Lopez, J. C.; Mata, S.; Caminati, W. All five forms of cytosine revealed in the gas phase. Angew. Chem. Int. Ed. 2013, 52, 2331-2334.

(29) Frisch, M. J.; Trucks, G. W.; Schlegel, H. B.; Scuseria, G. E.; Robb, M. A.; Cheeseman, J. R.; Scalmani, G.; Barone, V.; Mennucci, B.; Petersson, G. A.; Nakatsuji, H.; Caricato, M.; Li, X.; Hratchian, H. P.; Izmaylov, A. F.; Bloino, J.; Zheng, G.; Sonnenberg, J. L.; Hada, M.; Ehara, M.; Toyota, K.; Fukuda, R.; Hasegawa, J.; Ishida, M.; Nakajima, T.; Honda, Y.; Kitao, O.; Nakai, H.; Vreven, T.; Montgomery Jr., J. A.; Peralta, J. E.; Ogliaro, F. o.; Bearpark, M. J.; Heyd, J.; Brothers, E. N.; Kudin, K. N.; Staroverov, V. N.; Kobayashi, R.; Normand, J.; Raghavachari, K.; Rendell, A. P.; Burant, J. C.; Iyengar, S. S.; Tomasi, J.; Cossi, M.; Rega, N.; Millam, N. J.; Klene, M.; Knox, J. E.; Cross, J. B.; Bakken, V.; Adamo, C.; Jaramillo, J.; Gomperts, R.; Stratmann, R. E.; Yazyev, O.; Austin, A. J.; Cammi, R.; Pomelli, C.; Ochterski, J. W.; Martin, R. L.; Morokuma, K.; Zakrzewski, V. G.; Voth, G. A.; Salvador, P.; Dannenberg, J. J.; Dapprich, S.; Daniels, A. D.; Farkas, Ã. d. n.; Foresman, J. B.; Ortiz, J. V.; Cioslowski, J.; Fox, D. J., Gaussian 09, Revision D.01, Gaussian, Inc., 2009.

(30) Cruz-Cabeza, A. J.; Bernstein, J. Conformational polymorphism. Chem. Rev. 2014, 114, 2170-2191.

(31) Moellmann, J.; Grimme, S. DFT-D3 Study of Some Molecular Crystals. J. Phys. Chem. C $2014,118,7615-7621$. 
(32) Carter, D. J.; Rohl, A. L. Benchmarking Calculated Lattice Parameters and Energies of Molecular Crystals Using van der Waals Density Functionals. J. Chem. Theory Comput. 2014, $10,3423-3437$.

(33) Grimme, S.; Brandenburg, J. G.; Bannwarth, C.; Hansen, A. Consistent structures and interactions by density functional theory with small atomic orbital basis sets. J. Chem. Phys. $2015,143,054107$.

(34) G.M. Day, private communication.

(35) Brandenburg, J. G.; Alessio, M.; Civalleri, B.; Peintinger, M. F.; Bredow, T.; Grimme, S. Geometrical correction for the inter- and intramolecular basis set superposition error in periodic density functional theory calculations. J. Phys. Chem. A 2013, 117, 9282-9892.

(36) Nyman, J.; Pundyke, O. S.; Day, G. M. Accurate force fields and methods for modelling organic molecular crystals at finite temperatures. Phys. Chem. Chem. Phys. 2016, 18, 1582815837.

(37) Nyman, J.; Day, G. M. Static and lattice vibrational energy differences between polymorphs. CrystEngComm 2015, 17, 5154-5165.

(38) Alcobé, X.; Estop, E.; Aliev, A. E.; Harris, K. D. M.; Rodríguez-Carvajal, J.; Rius, J. Temperature-Dependent Structural Properties of $p$-Diiodobenzene: Neutron Diffraction and High-Resolution Solid State ${ }^{13}$ C NMR Investigations. J. Solid State Chem. 1994, 110, 20-27.

(39) Hongo, K.; Watson, M. A.; Iitaka, T.; Aspuru-Guzik, A.; Maezono, R. Diffusion Monte Carlo Study of Para-Diiodobenzene Polymorphism Revisited. J. Chem. Theory Comput. 2015, $11,907-917$.

(40) Verevkin, S. P.; Emel'yanenko, V. N.; Varfolomeev, M. A.; Solomonov, B. N.; Zherikova, K. V.; Melkhanova, S. V. Thermochemistry of dihalogen-substituted benzenes: data 
evaluation using experimental and quantum chemical methods. J. Phys. Chem. B 2014, 118, 14479-14492.

(41) Hulme, A. T.; Price, S. L.; Tocher, D. A. A new polymorph of 5-fluorouracil found following computational crystal structure predictions. J. Am. Chem. Soc. 2005, 127, 1116-1117.

(42) Amaral, L. M. P. F.; Szterner, P.; Miranda, M. S.; Ribeiro da Silva, M. A. V. Enthalpy of formation of 5-fluoro-1,3-dimethyluracil: 5-Fluorouracil revisited. J. Chem. Thermodyn. 2014, $75,106-115$

(43) Sancho-Garcia, J. C.; Pérez-Jiménez, A. J.; Olivier, Y. Determining the cohesive energy of coronene by dispersion-corrected DFT methods: Periodic boundary conditions vs. molecular pairs. J. Chem. Phys. $2015,142,054702$.

(44) Roux, M. V.; Temprado, M.; Chickos, J. S.; Nagano, Y. Critically Evaluated Thermochemical Properties of Polycyclic Aromatic Hydrocarbons. J. Phys. Chem. Ref. Data 2008, 37, 1855-1996.

(45) Thomas, S. P.; Spackman, M. A. The polymorphs of ROY: A computational study of lattice energies and conformational energy differences. Aust. J. Chem. 2018, 71, in press. 